\title{
The lubricants' parameters monitoring and data collecting
}

\author{
Gavril Grebenişan ${ }^{1, *}$, Nazzal Salem², and Sanda Bogdan ${ }^{3}$ \\ ${ }^{1}$ University of Oradea, Faculty of Manag. and Technological Eng., Industrial Engineering Department, Universitatii 1, Romania \\ ${ }^{2}$ Zarqa University, Faculty of Engineering Technology, P.O. Box 132222 Zarqa 13132, Jordan \\ ${ }^{3}$ University of Oradea, Faculty of Manag. and Techn. Eng., Mechanical Eng. and Automotive Department, Universitatii 1, Romania
}

\begin{abstract}
This approach is focused on Machine Intelligence for Diagnosis Automation, a research program, which promotes «preventative maintenance in manufacturing plants through the development of a fully automated prototype for oil analysis and fault prediction. The prototype is based on Artificial Intelligence (A.I.) software and online hardware ». Monitoring the condition of lubricants requires the examination of the physical, chemical and additive states, which maintain the quality of the lubricants, which is necessary for the proper functioning of the equipment. A lubricant monitoring program, especially from a qualitative point of view, will need to focus on both machine tool wear and degradation of lubricants, as well as on detecting and describing abnormal working conditions for both lubricants and machine parts. This goal can be satisfied by examining all the oils used in a company by completing laboratory tests to generate steps and acceptance classes, as well as unplanned contingency analyzes. These laboratory tests can be concentrated and classified into technology-based data sheets based on test-based information and test results, ultimately constituting consistent databases needed to generate monitoring and prevention reports. Data on the condition of the oil parameters used in the hydraulic system for lubricating machine tools have been collected during six months. The data as matrix organized, with 258648 rows (observations) and 21 columns (parameters).
\end{abstract}

\section{Introduction}

One of the major causes of premature wear of machines parts, moving or working, under load on the contact surface is the practice of improper lubrication. This includes contamination with foreign particles, the use of improper lubricants, the use of lubricants in excessive quantities or in insufficient quantities. In addition to the quality of lubricants to ensure technological processes under friction, wear and temperature control, lubricants also become diagnostic tools. A lubricant status monitoring program can provide information on additive inefficiency, change in viscosity, water content, coolant, and emulsion losses, and particulate matter contamination [1], [2]. Depending on the objectives and size of the monitoring system with the lubricant analysis, one can observe: control and analysis of the contaminating fluids; fluid properties analysis, metal wear analysis. Typical tests are:

- Number of foreign particles;

- Viscosity;

- Water content;

- Spectroscopy of chemical elements;

- Infrared Spectroscopy (FTIR);

- Measurement of Total Acid Number;

- Wear density (excluding measured particles);

- Analytical Ferrography (exception of the density of wear);

One of the key elements in the lubricant analysis is the ISO Code (ISO 4406). This code for solid contaminants expressly provides for the purity levels of liquids and is internationally recognized. The code provides a reference: the number of particles per volume unit, between 5 and 15 microns.

Table 1. The ISO codes.

\begin{tabular}{|c|c|c|}
\hline \multirow{2}{*}{$\begin{array}{c}\text { Range } \\
\text { Code }\end{array}$} & \multicolumn{2}{|c|}{ Particles per milliliter } \\
\hline & $\begin{array}{l}\text { More } \\
\text { than }\end{array}$ & $\begin{array}{c}\text { Up to / } \\
\text { Including }\end{array}$ \\
\hline 24 & 80000 & 160000 \\
\hline 23 & 40000 & 80000 \\
\hline 22 & 20000 & 40000 \\
\hline 21 & 10000 & 20000 \\
\hline 20 & 5000 & 10000 \\
\hline 19 & 2500 & 5000 \\
\hline 18 & 1300 & 2500 \\
\hline 17 & 640 & 1300 \\
\hline 16 & 320 & 640 \\
\hline 15 & 160 & 320 \\
\hline 14 & 80 & 160 \\
\hline 13 & 40 & 80 \\
\hline 12 & 20 & 40 \\
\hline 11 & 10 & 20 \\
\hline 10 & 5 & 10 \\
\hline 9 & 2.5 & 5 \\
\hline 8 & 1.3 & 2.5 \\
\hline 7 & 0.64 & 1.3 \\
\hline 6 & 0.32 & 0.64 \\
\hline
\end{tabular}

These two dimensions have some significance, because lower concentration gives an accurate appreciation of particle deposits in oil, and the upper level of 15 microns reflects the type of particle concentration

\footnotetext{
* Corresponding author: ggrebenisan@gmail.com
} 
from wear. The ISO code is represented by a combination of $R_{5 \text { microns }} / R_{15}$ microns, according to the reference data in the Table1.Thus, if the analysis resulted in the following data:-400 particles $>5$ microns / $\mathrm{ml}$; -65 particles $>15$ microns / $\mathrm{ml}$; then the ISO code is: ISO 16/13.

Contamination of lubricants with water is a subject of their analysis. The degree of contamination is expressed in PPM (parts per million) of water content. A percentage $(1 \%)$ of water is 10000 PPM. The low percentage of water in lubricants means good quality.

Viscosity is the critical property to follow in the case of lubricants. An increase in viscosity is more tolerable than a decrease in viscosity. Viscosity does not have an exact value; it varies within certain limits. This is guaranteed by the manufacturer.

The Neutralization Number, or the Total Acid Number (TAN), corresponds to its acidity. Acidity is primarily due to oil degradation. Acidity varies according to oil level.

Most experts in tribology recommend that oil analyzes be made mandatory for all new oils. These must include:

- Quantification of the number of particles in the workshop using porous sites (10 microns, characteristic mesh size);

- Determination of viscosity in the unit of work;

- Water content, using fluid separators;

Another set of laboratory analyzes should be performed on "critical" fluids, such as lubricants used in equipment tested at intervals of 60 to 90 days. Tests performed outside the workshop are:

Karl Fischer Specialized Laboratory Test;

- $\quad$ Special Spectroscopy Test of Elements;

- $\quad$ Infrared Spectroscopy (FTIR);

- Total Acid Number (TAN).

New oils must have an ISO code as well as a water content equal to or better than prescribed in the technical documentation of the machine tool.

Efficiency and cost-effectiveness calculations have highlighted the savings of up to $10 \%$ of operating costs if contaminated oils are changed in time, i.e., before the affected equipment is introduced into the service, repair or repair process. Regarding the benefits of investment and reliability, one can say that the key elements of a program for the monitoring, evaluation, and analysis of the state of the oils in a company with specific machining are:

- Acceptance criteria for new lubricants

- Acceptance criteria for servicing oils

- Defining storage conditions

- Clear storage procedures

- Handling and transport procedures

- Oil change procedures

- $\quad$ Ensure the filtering process

- Ensure the filtering process on the tool machine

- $\quad$ Specific airing conditions

Worldwide, the yearly damage to machinery due to wear is equivalent to the destruction of about $20 \%$ of their total. Tribology, science dealing with the study of the phenomena and processes of friction, lubrication and wear occurring in the superficial surfaces of the machine organs in contact with relative motion, seeks to prolong the duration of operation of machines and installations by combating or eliminating wear, both by knowing the causes (friction phenomenon) and by preventing or reducing wear by using proper lubrication.

Some preliminary considerations on how to behave in a relative movement of the machine parts with direct reference to the immediate surroundings of the couples formed in the manufacturing process and machine tools:

After surface lubrication, the friction may be:

- dry - in case of direct contact between the two coupling elements without lubrication (friction coefficient $\mu>0,3$ );

- $\quad$ at the limit (unctuous) - in this case, the interposition of molecular layers of lubricant occurs, the oil film reduces the contact between the elements of the coupling, but does not remove it, the lubrication exists but is at the limit $(0,1 \mu<0.3)$;

mixed (semifluid) - when the lubricating film has a thickness corresponding to the leakage of the fluids, but due to the roughness of the surfaces it breaks and recovers, at high frequencies, part of the normal load is taken over by the fluid film. Percentage, this assumption of normal forces, is estimated at several percentages, which are significant in the case of components with large, non-dimensional masses. In this case, the lubrication is called partial $(0.05<\mu$ $<0.1)$. This type of friction is encountered when starting and stopping sliding bearings, hydrostatic assembly of tightening assemblies, etc.

- $\quad$ fluid - when the joint surfaces of the coupler are separated, through a continuous lubricant film. The lubrication is fluid $(0.01<\mu<0.05)$.

It has been found in industrial manufacturing practice that most machine parts are not destroyed by both mechanical and thermal stresses, especially wear, due to sources of uncontrolled friction and inappropriate lubrication. Not always, inappropriate lubrication is due to design mistakes or non-conforming operations. These can also occur due to correct operation, but the composition of the machine tool materials in contact, as well as oils or lubricants, is inappropriate for various reasons. Some of these reasons may also be the deterioration of the properties and technical characteristics of the lubricants, but also of the materials of the machine parts in contact. By definition, the wear finds its physical expression in the loss of material on the contact surfaces of the relative moving bodies, resulting in the modification of the dimensions, the geometric shape and under certain temperature conditions, even leading to structural changes in the superficial layers [3] . The wear in lubricated friction contacts is influenced by:

\section{1 - Rheological lubrication regime}

The characteristic process values of the rheological lubrication regime are the gs viscosity parameter and the elasticity parameter ge:

$$
\begin{aligned}
& g_{v}=\frac{\alpha \cdot \omega}{R} \cdot\left(\frac{\omega}{\mu_{0} \cdot V}\right)^{\frac{1}{2}} \\
& g_{e}=\left(\frac{\omega}{E \cdot R}\right)^{\frac{1}{2}} \cdot\left(\frac{\omega}{\mu_{0} \cdot V}\right)^{\frac{1}{2}}
\end{aligned}
$$


in which: $\alpha$ is the viscosity-pressure coefficient; $\omega$ is the normal force per unit of contact width; $\mathrm{R}$ is the radius of curvature of the contact surface; E represents the effective elastic modulus; $\mu_{0}$ is the viscosity under normal conditions, and $\mathrm{V}$ is the relative speed between the contact surfaces [1-4]. The values of these parameters must be within the ranges: $g_{v}<1.5$, and $g_{e}<0.6$. If these conditions are not complied with due to the operating process, this is called the elastohydrodynamic lubrication process, characterized by the $g_{s}$ speed parameter and the $g_{l}$ load parameter, which must be within the following limits:

$$
\begin{gathered}
1.8<g_{s}=\alpha \cdot\left(\frac{E^{3} \cdot \mu_{0} \cdot V}{R}\right)^{\frac{1}{2}}<100, \\
1.0<g_{l}=\alpha \cdot\left(\frac{\omega \cdot E}{2 \cdot \pi \cdot R}\right)^{\frac{1}{2}}<100
\end{gathered}
$$

respectively.

\section{2 - Functional lubrication regime}

This is characterized by the minimum thickness of the oil film in the case of the pressed surfaces and is calculated by the formula:

$$
h_{0}=4.9 \cdot \frac{\mu_{0} \cdot V \cdot R}{\omega}
$$

where the coefficient " 4.9 " refers to a solid rigid with a lubricant isoviscosity. In the case of elastohydrodynamic conditions, the minimum thickness of the lubricant film shall be calculated with the formula:

$$
h_{0}=2.65 \cdot \frac{\alpha^{0.54} \cdot\left(\mu_{0} \cdot V\right)^{0.7} \cdot R^{0.43}}{\omega^{0.13} \cdot E^{0.03}}
$$

In the case of point-like contact of smooth surfaces, the minimum thickness of the lubricant film is:

$h_{0}=0.84 \cdot\left(\mu_{0} \cdot \alpha \cdot V\right)^{0.74} \cdot R^{0.41}(E / \omega)^{0.074}(5)$

If the process takes place by sliding the contacts on a thin film of lubricant, it is necessary that this regime is not considered a rheological one and the ratio lambda: $\lambda=$ $h_{0}{ }_{S}$, where $S=\frac{R_{1}+R_{2}}{2}=1.11 \cdot R_{a}$ and $R_{a}$, is the height of the surface roughness. If $\lambda>3$ then it can be considered that there is a fairly consistent likelihood that this contact of asperities is insignificant, so adhesive bonding is impossible to achieve. Otherwise, if $\lambda<1$, then the operating mode takes place at the extreme limit of lubrication, which implies the presence of wear and tear wear, respectively. Therefore, in this situation, lubrication conditions must be reconsidered. If this is practically impossible, the contact mode at the asperity limit must be determined by examining the plasticity index:

$$
\psi=\frac{E}{P_{y}} \cdot\left(\frac{\sigma}{\beta}\right)^{\frac{1}{2}}
$$

where E is the modulus of elasticity; $P_{y}$ is the normal pressure on the contact surface; $\beta$ - the asperity dispersion factor, $\sigma$-the mean standard deviation of the asperity height.

Thus, in the case of the mixed lubrication regime in which $1<\lambda<3$, in which most of the machine tools exhibit sliding or sliding / rolling contacts, the total load is divided between the asperity load and the lubricant film, respectively in this case only the load on the roughness contributes to the generation of wear. If $\psi<0.6$, contact between the asperities can be considered as elastic under any type of loading, and if this plasticity index $\psi>1$ then the contact can be considered partially elastic, even at low loading forces. If the plasticity index is in the range of 0.6 $<\psi<1$, the contact mode is a mixed one, and an increase in the loading force can generate a change of the elasticto-plastic contact. If $\psi<0.6$, there is a predominant metalmetal contact due to the fluctuation of the absorbed lubricant, and the idea of approaching the defects of the fractionated lubricant film should be considered.

\section{3 -The fractional lubricant film defect}

A property with measurable influence, which has a critical effect on wear in lubricated contacts, is the heat absorbed by the lubricant. This feature is valid in the case of adhesion, resulting, in practice, from direct metal-metal contact between roughness. If the lubricant molecules remain attached to the contact surfaces, then the probability of forming a prononced wear is extremely low. In physical terms, the film fractionation and the occurrence of fractionation defects of the oil film, respectively, $b$, can be defined as the ratio between the number of unrealized contacts, between the roughness, and the actual total number of contacts between the possible asperities:

$$
\beta=\frac{A_{m}}{A_{r}}
$$

in which $A_{m}$ represents the contact area, metal to metal, and $A_{r}$ represents the real contact area.

\section{4- Dispersion of loads in the contact}

For the discussion of this situation, it is considered a lubricated contact, in which only adhesion wear is present. The solution to the problem is based on the theory of partial elastohydrodynamic lubrication. According to this theory, both the contact between the asperities and the lubricant film contributes to the absorption of the dynamic loads. Thereby:

$$
W_{c}=W_{s}+W
$$

in which $W_{c}$ represents the total load on the contact surface, $W_{s}$ represents the charge taken up by the lubricant film, and $\mathrm{W}$ represents the load assumed by the asperities. According to experimental data, only part of the total load is considered responsible for adhesion. Analytically, the size of the contact pressure, empirically given by the relationship: 


$$
p=2 / 3 \cdot E \cdot(N r \sigma) \cdot(\sigma / r)^{\frac{1}{2}} \cdot F_{\frac{3}{2}}\left(d_{e} / \sigma\right)
$$

The total pressure resulting from the total load $W_{c}$ is given by:

$$
p_{c}=0.39 \cdot E^{\frac{2}{3}} \cdot W_{c}^{\frac{1}{3}} \cdot R_{e^{-\frac{2}{3}}}
$$

What determines the following ratio calculation:

$$
\begin{aligned}
& p / p_{c}=1.7 \cdot R_{e}^{\frac{2}{3}} \cdot W_{c}^{-\frac{1}{3}} \cdot E^{\frac{1}{3}}(N r \sigma) \cdot \\
& \cdot(\sigma / r)^{\frac{1}{2}} \cdot F_{\frac{3}{2}}\left(d_{e} / \sigma\right)
\end{aligned}
$$

in which $R_{e}^{\frac{2}{3}}$ is the relative radius of curvature of the contact surfaces, E- is the effective elastic modulus, Nthe relative density of the asperities, $r$ - is the mean radius of curvature of the asperity peak, $\sigma$ - is the standard deviation of the peaks, $F_{\frac{3}{2}}\left(d_{e} / \sigma\right)$ is a statistical function, and $d_{e}$ is the mean distance of separation between the tip of the asperities and the smooth surface.

The ratio between the lubricant pressure taken and the total pressure on the contact surfaces is given empirically by:

$$
p_{s} / p_{c}=\frac{1}{\lambda}\left(\frac{h_{0}}{\bar{h}}\right)^{6.3}
$$

where $\lambda$ is the specific thickness of the lubricating film, $h$ is the average height of the oil film in the case of rough surfaces, and $h_{0}$ is the height of the oil film in the case of smooth surfaces.

\section{5- A mathematical model of adhesion wear}

Theoretically, the amount of adhesion wearing should be expressed by a function of the metal to the metal contact surface and the slip distance:

$$
V=k_{m} \cdot A_{m} \cdot L
$$

Where $k_{m}$ is a non-dimensional constant specific to friction materials and independent of contaminants or lubricants. Expressing the actual contact surface, as a function of loading $\mathrm{W}$ and $\mathrm{P}$, the flow pressure, taking into account the oil film fractionation defects, $\beta$, obtains:

$$
V / L=k_{m} \cdot \beta \cdot(W / P)
$$

This equation has a special meaning for the terms it contains. Thus $k_{m}$ characterizes the tendency of shrinkage of the surfaces by wear, and the parameter $\beta$ indicates the ability of the lubricant to reduce the metal to the metal contact surface, taking values in the interval $[0,1]$.

\section{Operational Objective: Integration of the whole system, prototype development, testing}

The first of the directives of this operational objective is certainly to build a hardware system that can support the new requirements of the intended use range. In industrial practice, there is obviously a major requirement, namely that technology is structured so that it is "safe", without any risk to the work environment, and at the same time offers the opportunity to store and transmit in a way efficient information retrieved from sensors located on industrial equipment.

The designed system, which has begun this research, has some clear drawbacks, especially as regards the speed of adder acquisition, as well as its transmission and processing. This system was totally lacking in the possibility of data transmission, i.e., communication, of the local area networks(LAN) type, which revealed a fundamental deficiency in the intention of interfacing the system in the current industrial context. It was obvious that the system had to be rethought in terms of a new neural network approach, with the application of genetic algorithms, with the avoidance of traditional, high-risk switches or connectors. It was obvious the need to reorient the power supply to a mains or power source, max. 24 VDC, through LEMO connectors (fiber optic). It would have been desirable to involve an external touch screen, a smartphone model, through which the basic operations of the activation functions, display parameters, etc. could be performed. Considering these requirements, using specialized literature and specific search engines, a rigorous selection of appropriate component manufacturers was needed, so that the model of this system, which is prefigured, would allow a wide range of options and facilities to suit the intended purpose, and objectives listed.

A testing phase of a working model took place at the Mecoil (Italy) headquarters, in the company's lab, on a demo system with controlled parameters. The elements that were tested (physicochemical parameters) were evaluated in a first stage, on a single level. As the data was collected, based on predefined scenarios, "event / error modules" were interleaved on one level to test the system's response speed. The data was then processed and interpolated, at which point the contribution of "IA" became essential to evaluate the infinitesimal transformations (those that can escape the eye of a technician) of the multiparametric elements. The system was thus tested to detect between the " $n$ " elements to be taken into account simultaneously, and to evaluate in real time both the evolution and the trend of the monitored parameters, being "learned" to detect essential anomalies or possible failures. The overcoming of this early phase allowed the decision to install the system designed and physically installed on industrial installations (pilot systems) at EMSIL Techtrans SA-Romania, as well as in four other locations in Italy, to Mecoil partners, [6].

\subsection{Activities carried out}

In order to remove the obvious limitations shown by the first version of Monitoil's (Fig. 1.) monitoring system, it was decided to restructure the equipment by approaching a reasonable option for industrialization in the idea of efficient and logical improvement of the electronic components that would validate the project from the point 
of view of its operation under an environment of real, industrial thing.
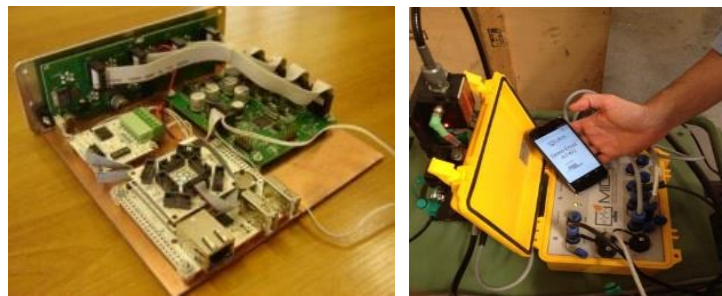

Fig. 1. Monitoring system Monitoil

Using advice with specialists in preventive maintenance, networking and robotics design (DM Electronics and Rodolfo Facchini), the planned activities were channeled towards the preliminary design of the acquisition board and the sensor work pattern, paying attention all possible causes of "distortion" of the signal and environmental and electrical interference. In this respect, consideration was given to the evaluation of a Raspberry type interface, with emphasis on data acquisition and sensing response checking. In addition, TecService Italy's TecService Italy consultancy has been used to build a first-ever version of the Machine Intelligence for Diagnosis Automation (MIDA), with both industrial and IP67 protection restrictions for these work environments, [6], (Fig. 2).

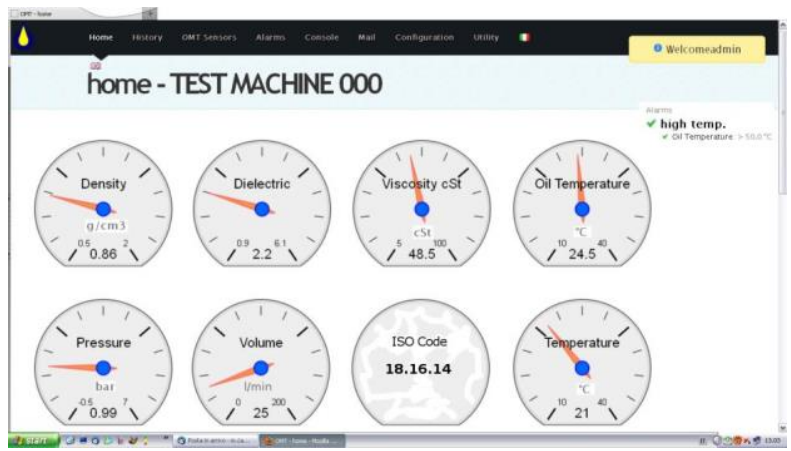

Fig. 2. Virtual Command Dashboard (www.mida-project.eu)

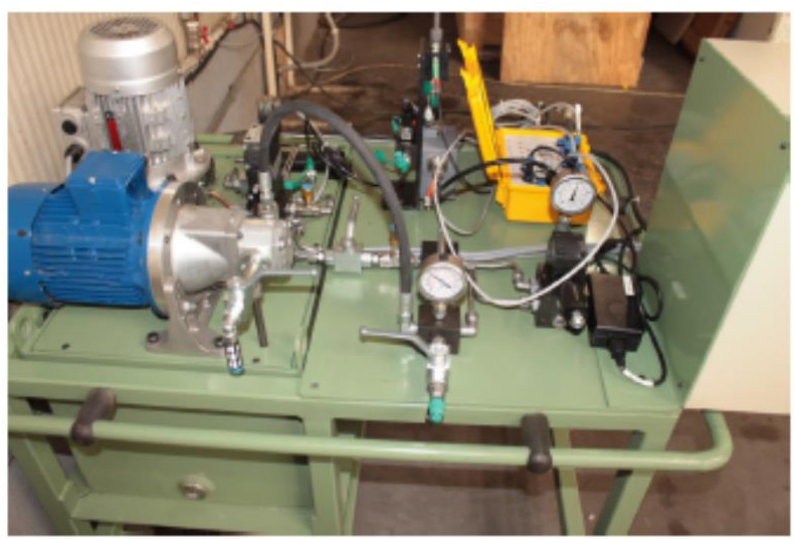

Fig. 3. The installation of MIDA on a machine tool at EMSIL Techtrans SA Oradea

Testing and tuning of the final phase algorithms were made possible by collecting and making available to the Project partners a set of observations data from the installation of MIDA on a machine at EMSIL Techtrans SA Oradea (Fig. 3.). Starting from the scheme of the first prototype, the Mida's miniseries were made. In addition to the test prototype test at EMSIL Techtrans SA Oradea, 4 other units were sent to Mecoil customers and partners to validate the device's operation under industrial conditions and obtain new test databases for artificial intelligence. The exploitation and testing of this equipment for many users simultaneously played an essential role in identifying problems, but also in evaluating the datalogger design phase(Fig. 4.).

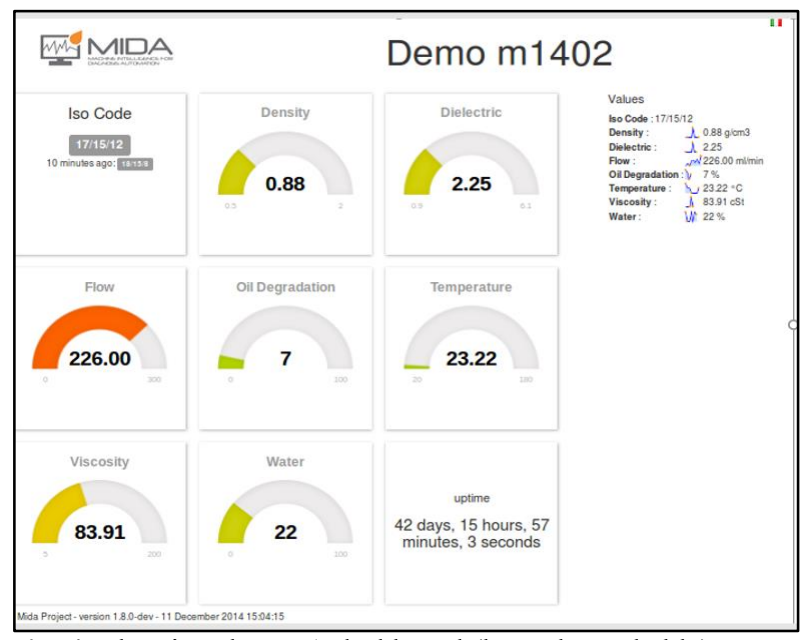

Fig. 4. The virtual MIDA dashboard (by web reachable)

\section{Results}

The data are matrix organized, with 258648 rows (observations) and 21 columns (parameters). The data refer to the analyzes made on the manufacturing line on the following parameters: density, viscosity, dielectric, water, oil degradation, temperature, ISO4, ISO6, ISO14, with obvious importance for the oil condition, but also for the operation of the industrial equipment being tested .

Particle sensors (Fig. 5) may be of several types: particle count or particle detector. The two are listed below:

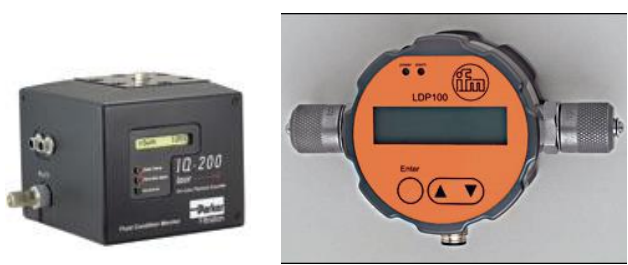

a)-particle counters

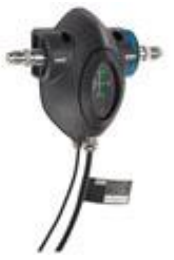

b)-particle detector

Fig. 5. Sensors used

Water sensors or moisture sensors can be of several types. Some are mounted on the pressure pit at others on the return circuit of the installations. Water or humidity sensors continuously monitor water / humidity in 
hydraulic and grease oils. They were designed especially for preventive maintenance of installations and machines. Reporting is done in $\%$ relative humidity of the water content, giving the user how close is the saturation point oil. Data on the condition of the oil parameters used in the hydraulic system for lubricating machine tools have been collected for six months. The data are matrixed, with 258648 rows (observations) and 21 columns (parameters). The parameters are presented in Table 2:

Table 2 The parameters collected

\begin{tabular}{|l|l|}
\hline id & measure identifier \\
\hline postgresql_timestamp & writing time \\
\hline time & writing time \\
\hline raw_data & $\begin{array}{l}\text { Maybe neglected, it is not } \\
\text { important (data before process } \\
\text { starts) }\end{array}$ \\
\hline icm_isocode & $\begin{array}{l}\text { ISO code } 4406 \text { (icm_iso4/ } \\
\text { icm_iso6/ icm_iso14) }\end{array}$ \\
\hline icm_rh & water saturation\% \\
\hline icm_flow & $\begin{array}{l}\text { oil flow through particle } \\
\text { counter }\end{array}$ \\
\hline icm_temp & $\begin{array}{l}\text { particle counter electronic } \\
\text { temperature }\end{array}$ \\
\hline icm_iso4 & $\begin{array}{l}\text { ISO } 4406 \text { code class particles } \\
>4 \mu m\end{array}$ \\
\hline icm_iso6 & $\begin{array}{l}\text { ISO } 4406 \text { code class particles } \\
>6\end{array}$ \\
\hline icm_iso14 & $\begin{array}{l}\text { ISO } 4406 \text { code class particles } \\
>14 \mu \mathrm{m}\end{array}$ \\
\hline icm_pc4 & number of particles $>4 \mu \mathrm{m}$ \\
\hline icm_pc6 & number of particles $>6 \mu \mathrm{m}$ \\
\hline icm_pc14 & number of particles $>14 \mu \mathrm{m}$ \\
\hline fps_vcst & viscosity [cSt] \\
\hline fps_v & viscosity [cP] \\
\hline fps_density & density \\
\hline fps_dielectric & dielectric constant \\
\hline fps_temp & oil temperature \\
\hline oh_temp & Oilhealth electronic temperature \\
\hline oh_parama & $\begin{array}{l}\text { Oilhealth paramA (not } \\
\text { important) }\end{array}$ \\
\hline oh_paramb & $\begin{array}{l}\text { Oilhealth paramB (not } \\
\text { important) }\end{array}$ \\
\hline oh_paramc & Oilhealth (not important) \\
\hline oh_od & Index of degradation OD\% \\
\hline Date & day of analysis \\
\hline time & hour/time of analysis \\
\hline & \\
\hline
\end{tabular}

Table 3. Data collected- the first 30/ 258648 rows and all columns of the database, are showed

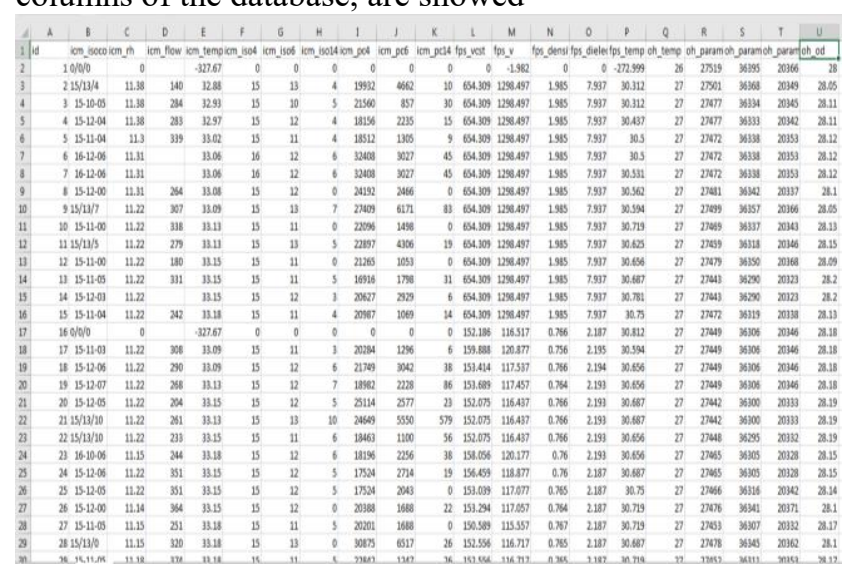

\section{Conclusion}

The activities carried out at this stage by the project partners were focused on the development and testing of prototype variants; analyzing continuous signals to identify the relevant signal characteristics and determining the useful data collection (Table 3) space to be able to provide an accurate estimate, or as close as possible to accuracy; Support Vector Machines training algorithms were developed to read the signal characteristics based on "learning". Numerical experiments have been conducted in different ways of using data through on-line analysis[5], [7]. Alternatively, the signal was divided into a larger number of frames that overlap partially (at the limit, NK-1 frames, provided that the first contains the data between $1 \ldots \mathrm{K}$, the second zone $2 \ldots \mathrm{K}+1$, etc.). In this way, the data is partially superimposed on a given order, but the advantage is that the data set becomes a time series of parameters in a smoother regression and therefore easier to extrapolate.

\section{Acknowledgment}

This work is supported by Machine Intelligence for Diagnosis Automation (MIDA), Research Program, National Program PN II, ERA MANUNET: NR 13081221/13.08.2013

\section{References}

1. Dennis H. Shreve-Integrated Condition Monitoring Technologies, IRD Balancing LLC, 2003.

2. Stolarski, T. A. -Tribology in Machine Design, Butterworth Heinemann, 1990.

3. Constantin, V., Palade, V.-Machine Tools Parts and Mechanisms (Organe de maşini, şi mecanisme), Ed. Fundaţiei Universitare Dunărea de Jos, Galaţi, 2004.

4. Prashad, H. -Solving tribology problems in rotating machines, Woodhead Publishing Ltd., Cambridge, 2006, England.

5. Grebenişan Gavril, Experimental research data processing by wavelet families transforms, Nonconventional Technologies Review (indexată ProQuest) - no. 1/2009, ISSN 14543087, Oradea, 2009.

6. G. Adriani, M. Campatelli, M. Paoli, -Monitoil ${ }^{\circledR}$ - Online sensors for efficient and cost-saving oil conditions' monitoring, Mecoil Diagnosi Meccaniche s.r.l., Florence, Italy, http://www.mecoil.net/wpcontent/uploads/2013/04/20120914-Articolocolonne Monitoil eng.pdf

7. Grebenişan Gavril, Sanda BogdanParameterized Finite Element Analysis of a Superplastic Forming Process, Using ANSYS, MATEC Web of Conferences, Volume 126, 2017, https://doi.org/10.1051/matecconf/201712603003 